\title{
AVALIAÇÃO DE CARACTERES QUANTITATIVOS RELACIONADOS COM O CRESCIMENTO VEGETATIVO ENTRE CULTIVARES DE CAFÉ ARÁBICA DE PORTE BAIXO $\left({ }^{1}\right)$
}

\author{
ZÉLIA MARIA TRAVASSOS SARINHO DE FREITAS $\left({ }^{2}\right)$; FRANCISCO JOSÉ DE OLIVEIRA $\left({ }^{3 *}\right)$; \\ SAMUEL PEREIRA DE CARVALHO $\left({ }^{4}\right)$; VENÉZIO FELIPE DOS SANTOS $\left({ }^{5}\right)$; \\ JOSÉ PEROBA DE OLIVEIRA SANTOS $\left({ }^{5}\right)$
}

\begin{abstract}
RESUMO
O objetivo deste trabalho foi estudar associação entre caracteres quantitativos relacionados com o crescimento vegetativo em cultivares de porte baixo de café arábica (Coffea arabica L.). O experimento foi desenvolvido na Unidade Experimental da Empresa Pernambucana de Pesquisa Agropecuária (IPA), município de Brejão (PE), localizado na mesorregião do agreste e na microrregião de Garanhuns, no ano agrícola de 2004/2005. O delineamento usado foi em blocos ao acaso, com quatro blocos e doze tratamentos constituídos por cultivares de porte baixo. Os caracteres foram avaliados através da análise de variância, estimativa dos parâmetros dos componentes da covariância e determinismo genético e dos coeficientes de correlações. Os resultados detectaram nas cultivares estudadas diferenças para altura das plantas e comprimento dos ramos palagiotrópicos. As cultivares Topázio MG 1189, Catucaí, Obatã IAC 1669-20 e Topázio MG 1190 são as melhores em desenvolvimento nos 12 primeiros meses de crescimento. O diâmetro de caule está fortemente correlacionado positivamente com o comprimento de ramos plagiotrópicos quando avaliada o incremento de crescimento. Quando avaliada aos 12 meses, o caráter altura de plantas correlacionou-se negativa e altamente significativa com o diâmetro de copa e o comprimento dos ramos plagiotrópicos, negativamente a número de ramos plagiotrópicos secundários e positivamente com o número de internódios de ramos plagiotrópicos.
\end{abstract}

Palavras-chave: Coffea arabica, variabilidade genética, caracteres correlacionados, pré-seleção juvenil.

\section{ABSTRACT \\ EVALUATION OF QUANTITATIVE TRAITS RELATED WITH THE VEGETATIVE GROWTH AMONG ARABICA COFFEE CULTIVARS}

The objective of this study was to verify the relationship between quantitative traits related with the vegetative growth in cultivars of short height of arabic coffee. The experiment was carried out in the Experimental Station of IPA (Research Institute of the State of Pernambuco), located in the town of Brejão, during in the agricultural year of 2004/2005. The experimental design used was randomized blocks, with four replications and twelve treatments, with each replications composed by 24 plants, disposed in

( ${ }^{1}$ Trabalho realizado com recursos da Empresa Pernambucana de Pesquisa Agropecuária (IPA). Recebido para publicação em 22 de março de 2006 e aceito em 12 de janeiro de 2007.

( ${ }^{2}$ ) Empresa Pernambucana de Pesquisa Agropecuária - IPA - Avenida San Martins, 1371, Bongi, Caixa Postal, 1022, 50761-000 Recife (PE), E-mail: zelia@ipa.br.

(3) Departamento de Agronomia, Universidade Federal Rural de Pernambuco, Rua Dom Manoel de Medeiros, n/s - Dois Irmãos, 52171-900 Recife (PE). E-mail: franseol@uol.com.br $\quad\left(^{*}\right)$ autor correspondente.

$\left({ }^{4}\right)$ Departamento de Agricultura, Universidade Federal de Lavras, Caixa Postal 3037, 37200-000 Lavras (MG). E-mail: samuelpc@ufla.br

$\left({ }^{5}\right)$ Empresa Pernambucana de Pesquisa Agropecuária, IPA, Av. San Martins, 1371, Bongi, Caixa Postal 1022, 50761-000 Recife (PE). E-mail: ipa@ipa.br 
three arrays, with useful area formed by the central array. The spacing used was $2.0 \mathrm{~m}$ between rows and $1.0 \mathrm{~m}$ among plants. The characters were appraised through the variance analysis, estimate of the genetic determinism and the coefficients of correlations. The results detected in the population studied differences among cultivars for height of plants and length of plagiotropic branches. The cultivars Topázio MG 1189, Catuaí Amarelo, Obatã IAC 1669-20 and Topázio MG 1190 were better in growing at 12 months. The stem diameter is strong and positively correlated with length of plagiotropic branches for growth rate. When evaluated at 12 months, it was correlated and negative and highly with the canopy diameter and the length of plagiotropic branches was correlated negatively to number of secondary branches and positively with number of nodes of plagiotropic branches.

Key words: Coffea arabica, genetic variability, correlated traits, juvenile pre-selection.

\section{INTRODUÇÃO}

A cafeicultura brasileira tem experimentado melhorias, decorrentes de pesquisas realizadas por várias instituições do país que se dedicam a este fim, principalmente na área de melhoramento e desenvolvimento de novas cultivares mais adaptadas às diferentes condições de cultivo, de alto padrão genético e elevado potencial de produção. O café também tem se destacado como fonte de renda para alguns agricultores no Agreste do Estado de Pernambuco, porém esses cultivadores têm escolhido as cultivares sem critério técnico, necessitando indicar as melhores sob aspecto de desenvolvimento vegetativo que sustente uma produtividade maior.

Os programas de melhoramento do cafeeiro no Brasil são de importância fundamental como fator de aumento da produção, da produtividade e de desenvolvimento socioeconômico das regiões de cultivo. Tais programas têm sido desenvolvidos com êxito para espécie Coffea arabica (FAzuoli, 1986; Carvalho e Fazuoli, 1993; Sera, 1998; SakiYama et al., 1999; Mendes, 1999). Objetivando o melhoramento do cafeeiro, a altura de planta tem sido usada como importante critério de seleção, preferindo-se as plantas mais baixas, que facilitam os tratos culturais e a colheita, além do que, em sistemas de plantio adensado, as variedades de porte baixo são preferidas devido a sua produtividade maior por área cultivada quando comparada com cultivares de porte alto (SAKIYAMA et al., 1999).

A avaliação do potencial produtivo de café de forma indireta tem sido investigada por diversos autores visando aumentar a eficiência na seleção. Correlações positivas com produtividade foram observadas com, por exemplo, diâmetro da copa (DHALIWAL, 1968; SILVAROLLA et al., 1997) e altura da copa (WALYARO e VAN DER VOSSEN, 1979). A quantificação e o conhecimento da natureza das correlações entre a produtividade e características morfológicas podem ser úteis no processo de seleção de cafeeiros (DHALIWAL, 1968).

Estimativas individuais de parâmetros genéticos, como a herdabilidade individual, são raras em café, citando-se apenas as obtidas por WALYARO e VAN DER Vossen (1979), no Quênia, e CiLAs et al. (1998), em Camarões, ambos para C. arabica, Leroy et al. (1994), na Costa do Marfim, e CiLAs et al. (2000), na Costa do Marfim e Togo, ambas para C. canephora. Estimativas de parâmetros genéticos e fenotípicos, em nível de médias de linhagens são comuns no Brasil, podendo-se citar Sera $(1980,1987)$, Sera e Alves (1999) e RESENDE et al. (2001).

Adẽo (2002), avaliando 42 cultivares de cafeeiro na fase adulta em Lavras (MG), observou correlação genotípica significativa entre a altura da planta e o diâmetro da copa $\left(\mathrm{r}_{\mathrm{G}}=0,774\right)$. Dias (2002), avaliando o crescimento vegetativo de 25 cultivares de café arábica, em Lavras (MG), encontrou resultados similares. FREITAS (2004), avaliando cinco caracteres fenológicos de 19 cultivares de café arábica no município de Brejão (PE), verificou magnitudes para as correlações genotípicas entre o diâmetro de caule e os caracteres altura da planta, número de ramos plagiotrópicos, comprimento dos ramos plagiotrópicos e número de internódios. Também, observou correlações genótipicas entre altura da planta e comprimento dos ramos plagiotrópicos; número de ramos plagiotrópicos entre comprimento dos ramos plagiotrópicos e número de internódios e entre comprimento dos ramos plagiotrópicos e número de internódios. O objetivo deste trabalho foi estudar a variabilidade e as associações entre os caracteres quantitativos relacionados com o crescimento vegetativo em cultivares de cafeeiros de porte baixo.

\section{MATERIAL E MÉTODOS}

O experimento foi desenvolvido na Unidade Experimental da Empresa Pernambucana de Pesquisa Agropecuária (IPA), município de Brejão (PE), localizado na mesorregião do agreste e na microrregião de Garanhuns, no ano agrícola de 2004/2005, em ambiente com altitude, de $788 \mathrm{~m}$, temperatura média anual de $22,15^{\circ} \mathrm{C}$, precipitação pluvial anual de 909,2 $\mathrm{mm}$ e déficit hídrico anual de $174 \mathrm{~mm}$ correspondente aos meses de novembro, dezembro e janeiro, no cenário 
de precipitação pluvial regular (VAREJÃo-SILVA, 2006). $\mathrm{O}$ delineamento usado foi em blocos ao acaso, com quatro repetições e doze tratamentos constituídos por cultivares de porte baixo de café (Coffea arabica L.) Topázio MG 1189, Catucaí Amarelo, Obatã IAC 166920, Topázio MG 1190, Rubi MG 1192, Catuaí Amarelo IAC 62-148, Catuaí Amarelo IAC 66, Catuaí Vermelho IAC 144, Tupi IAC 1669-33, Katipó, Catuaí Vermelho IAC 99 e Catuaí Vermelho IAC 81. Cada parcela foi composta por 24 plantas, dispostas em três fileiras, com área útil formada pela fileira central. O espaçamento foi de 2,0 m entre fileiras e 1,0 m entre covas, com uma planta por cova.

As observações foram efetuadas aos 6 e 12 meses (incremento de crescimento) após o plantio definitivo das mudas no campo, para caracteres (1) altura da planta (AP), medida em centímetros, do nível do solo até a gema apical do ramo ortotrópico das plantas; (2) número de ramos plagiotrópicos (NRP), realizada a contagem direta na planta em cada parcela, considerando-se número médio; (3) comprimento dos ramos plagiotrópicos (CRP), medido em centímetros e expresso em valores médios, no mesmo ramo selecionado para a medida do NRP; (4) número de internódios dos ramos plagiotrópicos (NIRP), contados em todos os ramos selecionados para a medida do CRP e (5) diâmetro do caule (DCA), medido em milímetros a 10 centímetros do solo utilizando o paquímetro digital. Aos 12 meses de idade das plantas foram também efetuadas, além dos caracteres citados anteriormente, o número de ramos plagiotrópicos secundários (NRPS), contados a partir dos ramos selecionados para a medida dos CRP e o diâmetro da copa (DCO), medido em centímetros a dois terços da copa. As análises de variância para todas as variáveis estudadas foram realizadas conforme VENCOVSKY e BARRIGA (1992). Foi utilizado o teste $F$ para determinar a significância dos tratamentos. As médias de tratamentos foram comparadas pelo teste de ScotT e KNOTT (1974). As estimativas dos parâmetros genéticos, componentes de variância e o determinismo genético das características, e dos coeficientes de correlações para os doze genótipos foram obtidas utilizando-se o Programa Genes (Cruz, 2003).

\section{RESULTADOS E DISCUSSÃO}

Pelo teste $\mathrm{F}$ aplicado, a análise de variância indicou diferenças altamente significativas $(\mathrm{P}<0,01)$ entre os genótipos para variável altura de plantas (AP) e comprimento dos ramos plagiotrópicos (CRP), evidenciando alto grau de variabilidade genética para essas características entre as cultivares nesse ambiente (Tabela 1).

$\mathrm{Na}$ tabela 2, constatam-se diferenças entre as cultivares em relação aos caracteres altura de plantas (AP) e comprimento dos ramos plagiotrópicos (CRP). A característica CRP é indicativo de diâmetro da copa e a combinação de AP e CRP junto com NRP é indicativo de maior número de nós produtivos, dois dos mais importantes componentes da produtividade (CARVALHO e Mônaco, 1967; Dhaliwal, 1968; Rocha et al., 1980; Vossen, 1985; SERA, 1987; Silvarolla et al., 1997; BONOMO et al., 2004; MiRANDA et al., 2005). As cultivares Topázio MG 1189, Catucaí Amarelo, Obatã IAC 1669-20 e Topázio MG 1190 são estaticamente mais altas, indicativo de maior número de ramos plagiotrópicos, confirmados em quatro das cinco de maior NRP.

Tabela 1. Esquema da análise de variância da diferença de crescimento das plantas no período de 6 e 12 meses de idade (incremento de crescimento) obtidos em cinco caracteres fenológicos, em doze genótipos de porte baixo de Coffea arabica L. Brejão (PE), 2006

\begin{tabular}{|c|c|c|c|c|c|c|}
\hline \multirow{2}{*}{ FV } & \multirow{2}{*}{ GL } & & & \multicolumn{3}{|c|}{ Quadrados Médios } \\
\hline & & $\mathrm{AP}$ & DCA & NRP & CRP & NIRP \\
\hline & & $\mathrm{cm}$ & $\mathrm{mm}$ & & $\mathrm{cm}$ & \\
\hline Blocos & 3 & 138,1206 & 6,9105 & 1,2821 & 26,2798 & 2,8015 \\
\hline Cultivares & 11 & $81,8806^{* *}$ & $3,3680^{\text {ns }}$ & $3,0696^{\mathrm{ns}}$ & $22,0116^{* *}$ & $0,8419^{\text {ns }}$ \\
\hline Resíduo & 33 & 27,7991 & 1,6975 & 3,1102 & 11,2481 & 0,8233 \\
\hline Total & 47 & - & - & - & - & - \\
\hline Médias & - & 36,7100 & 11,4621 & 15,1250 & 30,1849 & 10,0911 \\
\hline $\mathrm{CV}_{\mathrm{E}}(\%)$ & - & 14,3591 & 11,3671 & 11,6601 & 11,1109 & 8,9918 \\
\hline
\end{tabular}

\footnotetext{
${ }^{* *}$ Significativo ao nível de $1 \%$ de probabilidade pelo teste F. ns $:$ Não significativo. FV = fontes de variação; GL = grau de liberdade; CV $=$ coeficiente de variação experimental; $\mathrm{AP}=$ altura da planta; $\mathrm{DCA}=$ diâmetro do caule; $\mathrm{NRP}=$ número de ramos plagiotrópicos; $\mathrm{CRP}=$ comprimento dos ramos plagiotrópicos; NIRP = número de internódios nos ramos plagiotrópicos.
} 
A característica CRP é indicativo de diâmetro da copa e de maior número de nós produtivos por ramo; a combinação de AP e CRP é indicativo de maior número de nós produtivos por plantas, um dos mais importantes componentes da produtividade, a exemplo de Carvalho e Mônaco (1967), Dhaliwal (1968), Walyaro e Vossen (1979), Rocha et al. (1980), Vossen (1985), Sera (1987), Leroy et al. (1994), Silvarolla et al.(1997), Cilas et al. (1998) e Bonomo et al. (2004). Três das progênies mais desenvolvidas em altura são também as pertencentes ao grupo das progênies de maiores diâmetros indicados por CRP, "Catucaí Amarelo" (30,90 cm), “Obatã IAC 1669-20" $(32,60 \mathrm{~cm})$, “Topázio MG 1190" (32,31 cm). Esses caracteres podem auxiliar em seleção juvenil como indicado por SERA (1987). Esse fato está de acordo com os dados de número de internódios nos ramos plagiotrópicos (NIRP) que não diferem entre si, revelando que os de maior CRP têm proporcionalmente mais nós produtivos, fator decisivo para a produtividade por planta. A variação genética relativa à magnitude dos valores estimados para a maioria dos caracteres quanto ao incremento de caracteres vegetativos de 6 a 12 meses foram baixos, com exceção para altura de plantas (AP) (Tabela 3). $\mathrm{O}$ coeficiente de variação genética $\left(\mathrm{CV}_{\mathrm{G}}\right)$ oscilou de $0,6758 \%$ para NIRP e $10,0139 \%$ para AP, indicando a baixa magnitude da variabilidade genética para a característica incremento vegetativo entre as cultivares estudadas. Freitas (2004) observou resultado semelhante para o caráter altura de plantas. Contudo, a relação entre o coeficiente de variação genética $C_{G}$ e o coeficiente de variação experimental $\left(\mathrm{CV}_{\mathrm{E}}\right)$ mostrou valores inferiores a $1,0 \%$ evidenciando a condição desfavorável para AP $(0,70 \%)$, altamente desfavorável para DCA $(0,50 \%)$ e para CRP $(0,49 \%)$ e de inviabilidade para NIRP em termos de seleção. Os valores de coeficiente de determinação genotípica entre as médias das cultivares $\left(b_{m}\right)$ tiveram maiores possibilidades de sucesso na seleção em ordem decrescente $\mathrm{AP}(0,6605)$, DCA $(0,4960)$ e CRP $(0,4890)$. FREITAS (2004) obteve valor de herdabilidade para altura de plantas próximo de 0,7430 .

Pelos resultados da tabela 4 , verifica-se que houve diferença significativa $(P<0,05)$ entre os genótipos, aos 12 meses de idade, apenas para altura de planta (AP), indicando a existência de variabilidade entre os genótipos para esse caráter. Nas cultivares Topázio MG 1189, Catucaí Amarelo, Obatã IAC 1669-20, Topázio MG 1190 e Catuaí Vermelho IAC 144 (Tabela 5), observaramse as maiores alturas e não diferiram estatisticamente, entre si, para o carater altura de plantas (AP), mas diferiram dos outros genótipos testados. Nesse grupo, a cultivar Obatã IAC 1669-20 exibiu a maior altura de planta, indicando melhor desenvolvimento, complementado também por maiores números de ramos plagiotrópicos, indicativos de maior número de nós produtivos e, portanto, de um dos mais importantes caracteres correlacionados com a produtividade.

Tabela 2. Médias da diferença de crescimento das plantas no período de 6 e 12 meses de idade (incremento de crescimento) obtidas em doze genótipos de porte baixo de Coffea arabica L. e resultados do teste de ScotT e KNOTT a $5 \%$ de probabilidade. Brejão (PE), 2006

\begin{tabular}{|c|c|c|c|c|c|}
\hline \multirow{2}{*}{ Genótipos } & \multirow{2}{*}{$\mathrm{AP}$} & \multirow{2}{*}{ DCA } & \multicolumn{3}{|c|}{ Caracteres } \\
\hline & & & NRP & CRP & NIRP \\
\hline & $\mathrm{cm}$ & $\mathrm{mm}$ & & $\mathrm{cm}$ & \\
\hline Topázio MG 1189 & $43,59 \mathrm{a}$ & $10,89 \mathrm{a}$ & $16,56 \mathrm{a}$ & $26,25 \mathrm{~b}$ & $9,25 \mathrm{a}$ \\
\hline Catucaí Amarelo & $43,12 \mathrm{a}$ & $10,88 \mathrm{a}$ & $16,96 \mathrm{a}$ & $30,90 \mathrm{a}$ & 9,87 a \\
\hline Obatã IAC 1669-20 & $41,34 \mathrm{a}$ & $13,16 \mathrm{a}$ & 15,46 a & $32,62 \mathrm{a}$ & $10,75 \mathrm{a}$ \\
\hline Topázio MG 1190 & $41,34 \mathrm{a}$ & $11,27 \mathrm{a}$ & 15,21 a & 32,31 a & 9,90 a \\
\hline Rubi MG 1192 & $35,90 \mathrm{~b}$ & $11,51 \mathrm{a}$ & $14,28 \mathrm{a}$ & $29,65 \mathrm{a}$ & $10,06 \mathrm{a}$ \\
\hline Catuaí Amarelo IAC 62-148 & $35,15 \mathrm{~b}$ & $11,37 \mathrm{a}$ & $14,71 \mathrm{a}$ & $32,06 \mathrm{a}$ & $10,78 \mathrm{a}$ \\
\hline Catuaí Amarelo IAC 66 & $35,03 \mathrm{~b}$ & $11,31 \mathrm{a}$ & $14,28 \mathrm{a}$ & $30,43 \mathrm{a}$ & $10,00 \mathrm{a}$ \\
\hline Catuaí Vermelho IAC 144 & $35,00 \mathrm{~b}$ & $11,34 \mathrm{a}$ & $14,46 \mathrm{a}$ & $27,34 \mathrm{~b}$ & 10,18 a \\
\hline Tupi IAC 1669-33 & $34,43 \mathrm{~b}$ & $12,54 \mathrm{a}$ & $14,50 \mathrm{a}$ & $32,96 \mathrm{a}$ & $10,15 \mathrm{a}$ \\
\hline Katipó & $34,40 \mathrm{~b}$ & $12,58 \mathrm{a}$ & $15,50 \mathrm{a}$ & $31,06 \mathrm{a}$ & $10,15 \mathrm{a}$ \\
\hline Catuaí Vermelho IAC 99 & $35,25 \mathrm{~b}$ & 9,79 a & $14,90 \mathrm{a}$ & $26,50 \mathrm{~b}$ & 9,43 a \\
\hline Catuaí Vermelho IAC 81 & $30,00 \mathrm{~b}$ & $10,85 \mathrm{a}$ & $14,26 \mathrm{a}$ & $30,09 \mathrm{a}$ & $10,50 \mathrm{a}$ \\
\hline
\end{tabular}

Médias seguidas da mesma letra nas colunas não diferem significativamente, pelo teste de sCOTT e KNOTT a $5 \%$ de probabilidade. AP = altura da planta; $\mathrm{DCA}=$ diâmetro do caule; $\mathrm{NRP}=$ número de ramos plagiotrópicos; $\mathrm{CRP}=$ comprimento dos ramos plagiotrópicos; $\mathrm{NIRP}=$ número de internódios nos ramos plagiotrópicos. 
Tabela 3. Estimativas de análise de parâmetros genéticos para os caracteres altura de plantas (AP), diâmetro do caule (DCA), número de ramos plagiotrópicos (NRP), comprimento do ramo principal (CRP) e número de internódios no ramo principal (NIRP) obtidos em genótipos de porte baixo de Coffea arabica L. aos 6 e 12 meses de idade das plantas (incremento de crescimento). Brejão (PE), 2006

\begin{tabular}{lcccrc}
\hline \multirow{2}{*}{ Parâmetros genéticos } & \multicolumn{4}{c}{ Caracteres } \\
\cline { 2 - 6 } & AP & DCA & NRP & CRP & NIRP \\
\hline$\sigma_{F}^{2}$ & $\mathrm{~cm}$ & $\mathrm{~mm}$ & 0,7674 & 5,5029 & 0,2104 \\
$\sigma_{G}^{2}$ & 20,4701 & 0,8420 & - & 2,6909 & 0,0046 \\
$\sigma_{E}^{2}$ & 13,5203 & 0,4176 & 0,7775 & 2,8120 & 0,2058 \\
$c v_{G}(\%)$ & 6,9497 & 0,4243 & - & 5,4344 & 0,6758 \\
$c v_{e}(\%)$ & 10,0139 & 5,6380 & 11,6601 & 11,1109 & 8,9918 \\
$c v_{G} / c v_{e}$ & 14,3591 & 11,3671 & - & 0,4891 & 0,0751 \\
$b_{m}(\%)$ & 0,6973 & 0,4959 & - & 0,4890 & 0,2210 \\
\hline$\sigma_{F}$ & 0,6605 & 0,4960 & & & \\
\end{tabular}

$\sigma_{F}^{2}=$ variância fenotípica; $\sigma_{G}^{2}=$ variância genética; $\sigma_{E}^{2}=$ variância ambiental; $c v_{G}=$ coeficiente de variação genética; $C V_{\mathrm{e}}=$ coeficiente de variação experimental; $c v_{G} / c v_{e}=$ razão entre coeficiente de variação genética e coeficiente variação experimental; $b_{m}=c o e f i c i e n t e ~ d e$ determinação genotípica.

Tabela 4. Esquema da análise de variância dos dados obtidos em cinco caracteres fenológicos, em doze genótipos de porte baixo de Coffea arabica L. aos 12 meses de idade. Brejão (PE), 2006

\begin{tabular}{|c|c|c|c|c|c|c|c|c|}
\hline \multirow{2}{*}{ FV } & \multirow{2}{*}{ GL } & \multicolumn{7}{|c|}{ Quadrados Médios } \\
\hline & & $\mathrm{AP}$ & $\mathrm{DCO}$ & NRP & CRP & NIRP & NRRP & DCA \\
\hline & & \multicolumn{2}{|c|}{$-\mathrm{cm} \longrightarrow$} & & $\mathrm{cm}$ & & & $\mathrm{mm}$ \\
\hline Blocos & 3 & 0,0165 & 0,0376 & 4,8472 & 0,0054 & 1,6284 & 0,2428 & 10,6108 \\
\hline Cultivares & 11 & $0,0136^{* *}$ & $0,0158^{\mathrm{ns}}$ & $1,5123^{\text {ns }}$ & $0,0035^{\mathrm{ns}}$ & $1,5861^{\mathrm{ns}}$ & $0,11043^{\text {ns }}$ & $6,7262^{\text {ns }}$ \\
\hline Resíduo & 33 & 0,0038 & 0,0131 & 4,6701 & 0,0021 & 1,2676 & 0,9462 & 2,9674 \\
\hline Total & 47 & - & - & - & - & - & - & - \\
\hline Média & - & 0,7814 & 0,9587 & 25,0833 & 0,4971 & 16,2083 & 1,4949 & 19,8005 \\
\hline $\mathrm{CV}_{\mathrm{E}}(\%)$ & - & 7,9664 & 11,9672 & 8,6155 & 9,3189 & 6,9465 & 20,5782 & 8,6999 \\
\hline
\end{tabular}

${ }^{* *}$ Significativo ao nível de $1 \%$ de probabilidade pelo teste $F$. ${ }^{n s}$ Não significativo. $\mathrm{FV}=$ fontes de variação; $\mathrm{GL}=$ grau de liberdade; $\mathrm{CV} \mathrm{E}_{\mathrm{E}}=$ coeficiente de variação experimental; $\mathrm{AP}=$ altura da planta; $\mathrm{DCO}=$ diâmetro da copa; $\mathrm{NRP}=$ número de ramos plagiotrópicos; $\mathrm{CRP}=$ comprimento dos ramos plagiotrópicos; $\mathrm{NIRP}$ = número de internódios nos ramos plagiotrópicos; $\mathrm{NRPP}=$ número de ramos plagiotrópicos primários; $\mathrm{DCA}=$ diâmetro do caule.

Tabela 5. Médias de crescimento das plantas aos 12 meses de idade obtidas em doze genótipos de porte baixo de Coffea arabica L. e resultados do teste de SсOTT e KNOTT a 5\% de probabilidade. Brejão (PE). 2006

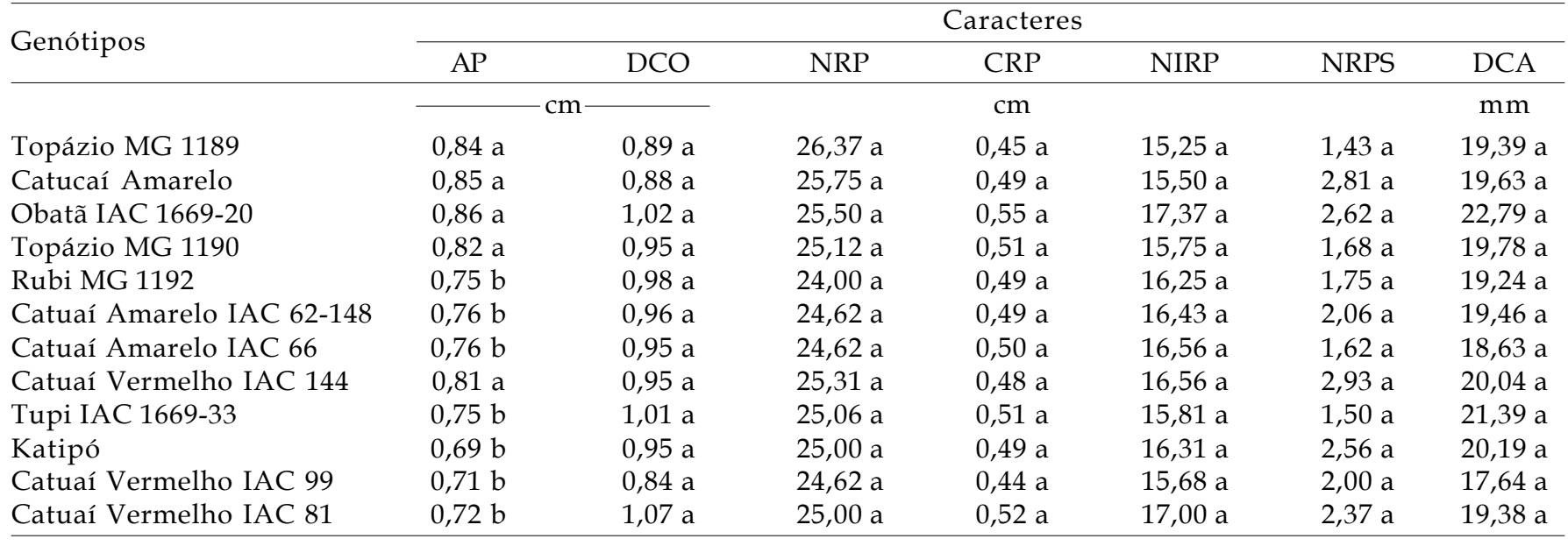

Médias seguidas da mesma letra nas colunas não diferem significativamente, pelo teste de scotT e кNOTT a $5 \%$ de probabilidade. AP $=$ altura de plantas; $\mathrm{DCO}=$ diâmetro da copa; $\mathrm{NRP}=$ número de ramos plagiotrópicos; $\mathrm{CRP}=$ comprimento dos ramos plagiotrópicos; NIRP = número de internódios nos ramos plagiotrópicos; NRPS = número de ramos plagiotrópicos secundários; DCA = diâmetro de caule. 
Verifica-se, de maneira geral, baixa variabilidade genética entre o material genético avaliado, conforme constatação dos coeficientes de variação genética e das herdabilidades médias. As variâncias genéticas, oscilando de 0,0003 para o comprimento dos ramos plagiotrópicos (CRP) a 0,0796 para número de internódios dos ramos plagiotrópicos (NIRP), indicam pequena variabilidade genética para todos os caracteres avaliados. A amplitude do coeficiente de variação genética foi de $3,8211 \%$ para CRP e $20,6820 \%$ para
DCA. A relação entre o coeficiente de variação genética $\mathrm{CV}_{\mathrm{G}}$ e o coeficiente de variação experimental $\left(\mathrm{CV}_{\mathrm{E}}\right)$ foi inferior a 1,0 evidenciando a condição desfavorável para a seleção dessas variáveis. Os coeficientes de determinação genotípica ao nível de médias de cultivares foram altos para a altura das plantas $\left(\mathrm{b}_{\mathrm{m}}=\right.$ $0,7164)$, mediano para CRP $\left(b_{\mathrm{m}}=0,4021\right)$ e DCO $\left(b_{\mathrm{m}}=\right.$ $0,5588)$ e baixo para DCA $\left(\mathrm{b}_{\mathrm{m}}=0,1673\right)$, NIRP $\left(\mathrm{b}_{\mathrm{m}}=\right.$ $0,2008)$ e NRPS $\left(b_{m}=0,1432\right)$ (Tabela 6$)$, indicando, respectivamente, altas, medianas e baixas possibilidades de sucesso na seleção.

Tabela 6. Estimativas de parâmetros genéticos para os caracteres altura da planta (AP), diâmetro do caule (DCA), número de ramos plagiotrópicos (NRP), comprimento do ramo principal (CRP), número de internódios no ramo principal (NIRP), número de ramos plagiotrópicos secundários (NRPS), obtidos em doze genótipos de porte baixo de café arábica (Coffea arabica L) obtidas aos 12 meses de idade. Brejão (PE), 2006

\begin{tabular}{|c|c|c|c|c|c|c|c|}
\hline \multirow{2}{*}{ Parâmetros genéticos } & \multicolumn{7}{|c|}{ Caracteres } \\
\hline & $\mathrm{AP}$ & DCA & NRP & CRP & NIRP & NRSP & $\mathrm{DCO}$ \\
\hline & $\mathrm{cm}$ & $\mathrm{mm}$ & & $\mathrm{cm}$ & & & $\mathrm{cm}$ \\
\hline$\sigma_{F}$ & 0,0034 & 0,0039 & 0,3780 & 0,0008 & 0,3965 & 0,0276 & 1,6815 \\
\hline$\sigma_{G}^{2}$ & 0,0024 & 0,0006 & - & 0,0003 & 0,0796 & 0,0039 & 0,9397 \\
\hline$\sigma_{E}^{2}$ & 0,0009 & 0,0032 & 1,1675 & 0,0005 & 0,3169 & 0,0236 & 0,7418 \\
\hline$c \mathcal{V}_{G(\%)}$ & 6,3305 & 20,6820 & - & 3,8211 & 1,7410 & 4,2063 & 4,8957 \\
\hline$C \mathcal{V}_{e}(\%)$ & 7,9664 & 11,9672 & - & 9,3189 & 6,9465 & 20,5782 & 8,6999 \\
\hline$c v_{G} / c v_{e}$ & 0,7946 & 0,0041 & - & 0,4100 & 0,2506 & 0,2044 & 0,5627 \\
\hline $\boldsymbol{b}_{m}(\%)$ & 0,7164 & 0,1673 & - & 0,4021 & 0,2008 & 0,1432 & 0,5588 \\
\hline
\end{tabular}

$\sigma_{F}^{2}=$ variância fenotípica; $\sigma_{G}^{2}=$ variância genética; $\sigma_{E}^{2}=$ variância ambiental; $c v_{G}=$ coeficiente de variação genética; $C V_{\mathrm{e}}=$ coeficiente de variação experimental; $c v_{G} / c v_{e}=$ razão entre coeficiente de variação genética e coeficiente variação experimental; $b_{\mathrm{m}}=$ coeficiente de determinação genotípica.

$\mathrm{Na}$ instalação de experimentos usando-se mudas, há diferenças ambientais maternais de tamanho e densidade das sementes resultando em diferenças de velocidade de germinação e crescimento na fase de viveiro. Essas diferenças podem estar se manifestando em maiores efeitos ambientais e, consequentemente, menor viabilidade genética nas avaliações, especialmente nas avaliações aos 12 meses. Apesar de mais trabalhosas, as avaliações de incremento de crescimento de 6 a 12 meses indicam ser mais precisos do que as avaliações aos 12 meses em termos de estimar a variabilidade ao nível de média de cultivares de porte baixo. A avaliação de incremento de crescimento reduz os efeitos de diferenças existentes no momento do plantio das mudas no campo. Adotando-se avaliações aos 12 meses somente se detecta variabilidade significativa por análise de variância para altura de planta (AP); para a avaliação por incremento de crescimento detectam-se diferenças significativas entre as cultivares para as características AP e CRP. As diferenças em determinismo genético está mais de acordo com o esperado relativo à análise de variância para avaliações por incremento de crescimento do que por avaliação aos 12 meses. A variabilidade para AP e CRP e, conseqüentemente, maior DCA, apesar de não significativo, com $b_{m}=0,6605,0,4890$ e 0,4960 , respectivamente, em contraste com $b_{\mathrm{m}}=0,7164,0,4021 \mathrm{e}$ 0,1673 para avaliações aos 12 meses.

Os coeficientes de correlação fenotípica $\left(\mathrm{r}_{\mathrm{F}}\right)$, genotípica $\left(r_{G}\right)$ e ambiental $\left(r_{E}\right)$ entre os caracteres $A P$, DCA, NRP, CRP e NIRP, resultante do incremento de crescimento vegetativo das plantas entre o período de 6 e 12 meses idade para as cultivares de café, de porte baixo, mostram que existe correlação genética entre o diâmetro do caule (DCA) e o comprimento dos ramos plagiotrópicos (CRP) positiva e altamente significativas a $1 \%$ de probabilidade de $0,9765^{* *}$ (Tabela 7). Logicamente, plantas de maior diâmetro de caule implicam plantas com maior comprimento de ramos plagiotrópicos e, conseqüentemente, maior diâmetro de copa. Medir o CRP é bem mais difícil do que avaliar o DCA aos 12 meses. Assim, há possibilidade de pré-selecionar aos 12 meses para maior produtividade fotossintética, avaliando o DCA com altas possibilidades de êxito com baixo custo. 
Tabela 7. Coeficientes de correlação fenotípica $\left(\mathrm{r}_{\mathrm{F}}\right)$, genotípica $\left(\mathrm{r}_{\mathrm{G}}\right)$ ambiental $\left(\mathrm{r}_{\mathrm{E}}\right)$ entre cinco caracteres altura da planta (AP), diâmetro do caule (DCA), número de ramos plagiotrópicos (NRP), comprimento dos ramos plagiotrópicos (CRP) e número de internódios nos ramos plagiotrópicos (NIRP), obtidos sete genótipos de porte baixo de Coffea arabica L. em plantas com 6 e 12 meses de idade (incremento de crescimento). Brejão (PE), 2006

\begin{tabular}{|c|c|c|c|c|c|c|}
\hline Caracteres & Correlações & $\mathrm{AP}$ & DCA & NRP & CRP & NIRP \\
\hline \multirow{5}{*}{$\mathrm{AP}$} & $\mathrm{r}$ & $\mathrm{cm}$ & $\mathrm{mm}$ & & $\mathrm{cm}$ & \\
\hline & $\mathrm{r}_{\mathrm{F}}$ & 1 & $0,1775^{\mathrm{ns}}$ & $0,7498^{* *}$ & $0,1116^{\mathrm{ns}}$ & $-0,2432^{\mathrm{ns}}$ \\
\hline & $r_{G}$ & 1 & $0,0284^{\mathrm{ns}}$ & - & $-0,1695^{\mathrm{ns}}$ & $-0,3221^{\mathrm{ns}}$ \\
\hline & $\mathrm{r}_{\mathrm{E}}$ & 1 & $0,3898^{*}$ & $0,3744^{*}$ & $0,4992^{* *}$ & $0,5682^{* *}$ \\
\hline & $\mathrm{r}_{\mathrm{F}}$ & - & 1 & $-0,0797^{n s}$ & $0,6712^{* *}$ & $0,5786^{* *}$ \\
\hline \multirow[t]{3}{*}{ DCA } & $r_{G}$ & - & 1 & - & $0,9765^{* *}$ & $0,2621^{\mathrm{ns}}$ \\
\hline & $\mathrm{r}_{\mathrm{E}}$ & - & 1 & $0,3536^{*}$ & $0,3750^{*}$ & $0,2512^{\mathrm{ns}}$ \\
\hline & $\mathrm{r}_{\mathrm{F}}$ & - & - & 1 & $-0,1112^{\mathrm{ns}}$ & $-0,3947^{*}$ \\
\hline \multirow[t]{3}{*}{ NRP } & $r_{G}$ & - & - & 1 & - & - \\
\hline & $\mathrm{r}_{\mathrm{E}}$ & - & - & 1 & $0,3007^{\mathrm{ns}}$ & $0,2457^{\mathrm{ns}}$ \\
\hline & $\mathrm{r}_{\mathrm{F}}$ & - & - & - & 1 & $0,6758^{* *}$ \\
\hline \multirow[t]{3}{*}{ CRP } & $r_{G}$ & - & - & - & 1 & $0,1554^{\mathrm{ns}}$ \\
\hline & $\mathrm{r}_{\mathrm{E}}$ & - & - & - & 1 & $0,6208^{* *}$ \\
\hline & $\mathrm{r}_{\mathrm{F}}$ & - & - & - & - & 1 \\
\hline \multirow[t]{2}{*}{ NIRP } & $r_{G}$ & - & - & - & - & 1 \\
\hline & $r_{E}$ & & - & - & - & 1 \\
\hline
\end{tabular}

${ }^{* *}$ e Significativo ao nível de $1 \%$ e $5 \%$ de probabilidade pelo teste t. ${ }^{\text {ns }}$ Não significativo.

$\mathrm{Na}$ tabela 8, estão representados os coeficientes de correlação entre alguns caracteres vegetativos de cultivares de café de porte baixo aos 12 meses de idade.

A correlação entre a altura de plantas e o diâmetro de copa $(-0,8102)$ revelou-se estatisticamente significativa, ou seja, quanto maior a altura das plantas menor o diâmetro de projeção de copas. Este fato revela que existe mecanismo de compensação de um crescimento vertical por um mecanismo horizontal. Assim, há possibilidade de pré-selecionar parcialmente aos 12 meses para altura de diâmetro da copa maior, selecionando-se para altura da planta menor e, assim, obter maior produtividade fotossintética.

O comprimento dos ramos plagiotrópicos (CRP) correlacionou-se geneticamente positivo e significativo $\left(0,4838^{* *}\right)$ com o número de internódios dos ramos plagiotrópicos (NIRP). O NIRP é bom indicador de número de nós produtivos de uma planta. O CRP é bom indicador de número de nós produtivos, um dos componentes principais da produtividade da planta, e pode ser usado como medida mais fácil do que contar o número de nós produtivos de uma planta. O número de nós produtivos de uma planta representa uma porção importante dessa planta, visto que tem uma alta possibilidade de sucesso em pré-selecionar aos 12 meses, indiretamente, com base em avaliação visual no campo para CRP.

O CRP correlacionou-se geneticamente positivo e altamente significativo $(0,8387)$ com o diâmetro do caule (DCA), o que está de acordo com a maior necessidade de sistema de transporte de seiva para sustentar um crescimento maior com diâmetro da copa da planta maior. Esse fato indica alta possibilidade de sucesso na pré-seleção juvenil para o diâmetro de caule, altamente correlacionada com o tamanho da copa e parte da produtividade.

A avaliação para CRP no campo é mais difícil do que a avaliação para DCA aos 12 meses. Se selecionada para o DCA poderá selecionar em boa parte para maior produtividade fotossintética.

A correlação entre CRP e NRPS. $\left(r_{G}=\right.$ $0,6724^{* *}$ ) revelou-se estatisticamente significativa. Um número de ramos plagiotrópicos secundários indica menor crescimento em CRP ou vice-versa. Assim, se selecionar para maior NRPS, em parte poderá selecionar indiretamente para menor CRP e, conseqüentemente para menor diâmetro da copa, o que poderá ser desejável para o plantio adensado. 
Tabela 8. Coeficientes de correlação fenotípica $\left(\mathrm{r}_{\mathrm{F}}\right)$, genotpica $\left(\mathrm{r}_{\mathrm{G}}\right)$ ambiental $\left(\mathrm{r}_{\mathrm{E}}\right)$ entre sete caracteres altura da planta $(\mathrm{AP})$, diâmetro do caule (DCA), número de ramos plagiotrópicos (NRP), comprimento do ramo principal (CRP), número de internódios no ramo principal (NIRP), número de ramos plagiotrópicos secundários (NRPS), obtidos em doze genótipos de porte baixo de café arábica (Coffea arabica L) aos 12 meses de idade. Brejão (PE). 2006

\begin{tabular}{|c|c|c|c|c|c|c|c|c|}
\hline Caracteres & Correlações & $\mathrm{AP}$ & $\mathrm{DCO}$ & NRP & CRP & NIRP & NRPS & DCA \\
\hline & $\mathrm{r}$ & 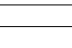 & 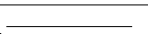 & & $\mathrm{cm}$ & & & $\mathrm{mm}$ \\
\hline \multirow{4}{*}{$\mathrm{AP}$} & $r_{F}$ & 1 & $-0,1285^{\mathrm{ns}}$ & $0,6745^{* *}$ & $0,1941^{\mathrm{ns}}$ & $-0,1204^{\mathrm{ns}}$ & $0,2139^{\mathrm{ns}}$ & $0,3105^{\mathrm{ns}}$ \\
\hline & $r_{G}$ & 1 & $-0,8102^{* *}$ & - & $-0,1455^{\mathrm{ns}}$ & $-0,1537^{\mathrm{ns}}$ & $0,4092^{*}$ & $0,1764^{\mathrm{ns}}$ \\
\hline & $r_{E}$ & 1 & $0,3127^{\mathrm{ns}}$ & $0,6237^{* *}$ & $0,6612^{* *}$ & $0,7836^{* *}$ & $0,1681^{\mathrm{ns}}$ & $0,5623^{* *}$ \\
\hline & $\mathrm{r}_{\mathrm{F}}$ & - & 1 & $-0,1957^{\mathrm{ns}}$ & $0,8137^{* *}$ & $0,7243^{* *}$ & $0,0943^{\mathrm{ns}}$ & $0,6410^{* *}$ \\
\hline \multirow[t]{3}{*}{$\mathrm{DCO}$} & $r_{G}$ & - & 1 & - & $0,0446^{\mathrm{ns}}$ & $0,2110^{\mathrm{ns}}$ & $-0,1031^{\mathrm{ns}}$ & $0,1810^{\mathrm{ns}}$ \\
\hline & $r_{E}$ & - & 1 & $0,6979^{* *}$ & $0,4117^{*}$ & $0,3314^{*}$ & $0,5816^{* *}$ & $0,4792^{*}$ \\
\hline & $\mathrm{r}_{\mathrm{F}}$ & - & - & 1 & $-0,0703^{\mathrm{ns}}$ & $-0,3058^{\mathrm{ns}}$ & $0,2192^{\mathrm{ns}}$ & 0,2038 \\
\hline \multirow[t]{3}{*}{ NRP } & $r_{G}$ & - & - & 1 & - & - & - & - \\
\hline & $r_{E}$ & - & - & 1 & $0,4884^{*}$ & $0,4983^{*}$ & $0,3295^{*}$ & $0,6451^{* *}$ \\
\hline & $\mathrm{r}_{\mathrm{F}}$ & - & - & - & 1 & $0,6774^{* *}$ & $0,2497^{\mathrm{ns}}$ & $0,7196^{* *}$ \\
\hline \multirow[t]{3}{*}{ CRP } & $r_{G}$ & - & - & - & 1 & $0,4838^{*}$ & $-0,6724^{* *}$ & $0,8387^{* *}$ \\
\hline & $r_{\mathrm{E}}$ & - & - & - & 1 & $0,7810^{* *}$ & $0,5744^{* *}$ & $0,6269^{* *}$ \\
\hline & $r_{F}$ & - & - & - & - & 1 & $0,4192^{*}$ & $0,5392^{* *}$ \\
\hline \multirow[t]{3}{*}{ NIRP } & $r_{G}$ & - & - & - & - & 1 & $0,6241^{* *}$ & $0,5554^{* *}$ \\
\hline & $r_{E}$ & - & - & - & - & 1 & $0,3787^{*}$ & $0,5947^{* *}$ \\
\hline & $\mathrm{r}_{\mathrm{F}}$ & - & - & - & - & - & 1 & $0,1848^{\mathrm{ns}}$ \\
\hline \multirow[t]{3}{*}{ NRRP } & $r_{G}$ & - & - & - & - & - & 1 & $-0,3705^{*}$ \\
\hline & $r_{E}$ & - & - & - & - & - & 1 & $0,4711^{*}$ \\
\hline & $r_{F}$ & - & - & - & - & - & - & 1 \\
\hline \multirow[t]{2}{*}{ DCA } & $r_{G}$ & - & - & - & - & - & - & 1 \\
\hline & $r_{E}$ & - & - & - & - & - & - & 1 \\
\hline
\end{tabular}

${ }^{* *} \mathrm{e}^{*}$ Significativo ao nível de $1 \%$ e $5 \%$ de probabilidade pelo teste $\mathrm{t}$. ${ }^{\text {ns }}$ Não significativo.

Assim, existe possibilidade de melhorar a eficiência de seleção para produtividade de grãos de café realizando a pré-seleção juvenil aos 12 meses de características correlacionadas com a produtividade, selecionando para a produtividade fotossintética que será a base da produtividade de grãos usando-se características que se manifestam aos 12 meses de campo.

\section{CONCLUSÕES}

1. Na população estudada foram detectadas diferenças estatísticas para a altura das plantas e comprimento dos ramos plagiotrópicos e existem possibilidades de sucesso na seleção juvenil aos 12 meses, baseada em determinismo genético para altura das plantas, comprimento de ramos plagiotrópicos e diâmetro do caule.

2. As cultivares Topázio MG 1189, Catucaí Amarelo, Obatã IAC 1669-20 e Topázio MG 1190 foram as melhores em desenvolvimento nos 12 primeiros meses de crescimento.

3. Para cultivares de porte baixo desta pesquisa, como critérios de pré-seleção juvenil, podem ser usadas a característica diâmetro do caule para produtividade fotossintética, a característica comprimento menor de ramos primários para maior ramificação secundária e menor altura para maior diâmetro da copa.

\section{REFERÊNCIAS}

ADÃO, W.A. Análise de cultivares do cafeeiro (Coffea arabica L.) por meio de características morfológicas e agronômicas. 2002. 59f. Dissertação (Mestrado em Fitotecnia) - Universidade Federal de Lavras, Lavras.

BONOMO, P.; CRUZ, C.D; VIANA, J.M.S.; PEREIRA, A.A.; OLIVEIRA, V.R.; CARNEIRO, P.C.S. Avaliação de progênies obtidas de cruzamentos de descendentes do Híbrido de Timor com as cultivares Catuaí Vermelho e Catuaí Amarelo. Bragantia, Campinas, v.63, n.2, p.207-219, 2004. 
CARVALHO, A.; FAZUOLI, L.C. Café. In: FURLANI, A.M.C.; VIEGAS, G.P.O.(Ed.) Melhoramento de plantas no Instituto Agronômico. Campinas: Instituto Agronômico, 1993. p.29-76.

CARVALHO, A.; MÔNACO, L.C. Melhoramento do cafeeiro. XXXI. Ensaios de populações $\mathrm{F}_{2}$ de híbridos entre cultivares de Coffea arabica. Bragantia, Campinas, v.26, n.5, p.79-92, 1967.

CILAS, C.; BOUCHARMONT, P.; BOCCARA, M.; ESKES, A.B.; BARADAT, P. Prediction of genetic value for coffee production in Coffea arabica from a half-diallel with lines and hybrids. Euphytica, Dordrecht, v.104, p.49-59, 1998.

CILAS, C.; MONTAGNON, C.; BERTRAND, B.; GODIN, C. Wood elasticity of several Coffea canephora clones. A new trait to be included in selection schemes. Agronomie, Paris, v.20, p.439-444, 2000.

CRUZ, C.D. Programa Genes: aplicativo computacional em genética e estatística. Viçosa: UFV. 2003.

DHALIWAL, T.S. Correlations between yield morphological characters in Puerto Rican and Columnaris varieties of Coffea arabica L. Journal of the Agricultural University of Porto Rico, Puerto Rico, v.52, p.29-37, 1968.

DIAS, F.P. Caracterização de progênies de cafeeiros (Coffea arabica L.) por meio de técnicas multivariadas. 2002. 64f. Dissertação (Mestrado em Fitotecnia) - Universidade Federal de Lavras, Lavras.

FAZUOLI, L.C. Genética e melhoramento do cafeeiro. In: RENA, A.B.; MLAVOLTA, E.; ROCHA, M.; YAMADA, T. Cultura do cafeeiro: fatores que afetam a produtividade. Piracicaba: Associação Brasileira para Pesquisa da Potassa e do Fosfato, 1986. p.87-113.

FREITAS, Z.M.T.S. Características fenológicas de cafeeiros (Coffea arabica L.) em pós-plantio no Agreste de Pernambuco. 2004. 52f. Tese (Doutorado em Fitotecnia) - Universidade Federal de Lavras, Lavras.

LEROY, T.; MONTAGNON, C.; CILAS, C.; CHARRIER, A.; ESKES, A.B. Reciprocal recurrent selection applied to Coffea canephora. II. Estimation of genetic parameters. Euphytica, Dordrecht, v.74, p.121-128, 1994.

MENDES, A.N.G. Métodos de melhoramento aplicados na cultura do cafeeiro. In: SIMPÓSIO DE ATUALIZAÇÃO EM GENÉTICA E MELHORAMENTO DE PLANTAS, 3., 1999, Lavras. Anais... UFLA, 1999. p.18-35.

MIRANDA, J.M.; PERECIN, D.; PEREIRA, A.A. Produtividade e resistência à ferrugem do cafeeiro (Hemileia vastatrix Berk. Et Br.) de progênies $\mathrm{F}_{5}$ de Catuaí Amarelo com o Híbrido de Timor. Ciência Agrotécnica, Lavras, v.29, n.6. p.1195-1200, 2005.

RESENDE, M.D.V. DE; FURLANI-JÚNIOR, E.; MORAES, M.L.T.DE; FAZUOLI, L.C. Estimativas de parâmetros genéticos e predição de valores genotípicos no melhoramento do cafeeiro pelo procedimento REML/BLUP. Bragantia, Campinas, v.60, n.3, p.185-193. 2001.
ROCHA, T.R.; CARVALHO, A.; FAZUOLI, L.C. Melhoramento do cafeeiro. XXXVIII. Observações sobre progênies do cultivar Mundo Novo de Coffea arabica L. Bragantia, Campinas, v.39, n.1, p.147-160, 1980.

SAKIYAMA, N.S.; PEREIRA, A.A.; ZAMBOLIM, L. Melhoramento do cafeeiro. In: BORÉM, A. (Ed.). Melhoramento de espécies cultivadas. Viçosa: UFV, 1999. p.189-204.

SCOTT, A. J.; KNOTT, M. A. A cluster analysis method for grouping means in the analysis of variance. Biometrics, Raleigh, v.30, n.3, p.507-512, 1974.

SERA, T. Desafios no melhoramento genético do cafeeiro. In: SIMPÓSIO DE ATUALIZAÇÃO EM GENÉTICA E MELHORAMENTO DE LANTAS, 2., 1998, Lavras. Anais...Lavras: UFLA, 1998. p.105-122.

SERA, T. Estimação dos componentes da variância e do coeficiente de determinação genotípica da produção de grãos de café. Piracicaba, 1980. 62p. Dissertação (Mestrado em Genética e Melhoramento de Plantas) - Escola Superior de Agricultura "Luiz de Queiroz", Universidade de São Paulo.

SERA, T. Possibilidades de emprego de seleção nas colheitas iniciais de café (Coffea arabica L. cv. Acaiá). Piracicaba, 1987. 147f. Tese (Doutorado em Genética e Melhoramento de Plantas) - Escola Superior de Agricultura "Luiz de Queiroz", Universidade de São Paulo.

SERA, T.; ALVES, S.J. Melhoramento genético de plantas perenes. In: DESTRO, D.; MONTALVÁN, R. (Ed.). Melhoramento Genético de Plantas. Londrina: Editora UEL, 1999. p.369-422.

SILVAROLLA, M.B.; GUERREIRO FILHO, O.; LIMA, M.A.L.; FAZUOLI, L.C. Avaliação de progênies derivadas do híbrido timor com resistência ao agente da ferrugem. Bragantia, Campinas, v.56, n.1, p.47-58, 1997.

VAREJÃO-SILVA, M.A. Processamento e resultados: software climatologia do Nordeste. Recife (PE), 2006. (Comunicação Pessoal)

VENCOVSKY, R.; BARRIGA, P. Genética biométrica no fitomelhoramento. Ribeirão Preto, Sociedade Brasileira de Genética, 1992. 494p.

VOSSEN, H.A.M. van der. Coffee selection and breeding. In: CLIFFORD, M.N.; WILSON, K.C. Coffee: botany, biochemistry and production of beans and beverage. Croom Helm, 1985, Cap. 3, p.48-96.

WALYARO, D.J.; VOSSEN, H.A.M. van der. Early determination of yield potential in arabic coffee by applying index selection. Euphytica, Dordrecht, v.28, p.465-472, 1979. 